M.Q. Du ${ }^{1}$, B.J. Tai ${ }^{1}$, H. Jiang ${ }^{1}$, E.C.M. Lo ${ }^{2}$ M.W. Fan ${ }^{3 *}$, and Z. Bian ${ }^{3 *}$

${ }^{1}$ Department of Preventive Dentistry, School of Stomatology, Wuhan University, Hubei, China; ${ }^{2}$ Faculty of Dentistry, University of Hong Kong, Hong Kong SAR, China; and ${ }^{3} \mathrm{Key}$ Lab for Oral Biomedical Engineering of Ministry of Education, School and Hospital of Stomatology, Wuhan University, Hubei, China; *corresponding authors, kqyywjtx@public.wh.hb.cn, fmwbgsh@sina.com.cn, hbcoh@public.wh.hb.cn

J Dent Res 85(6):557-559, 2006

\section{A Two-year Randomized Clinical Trial of Chlorhexidine Varnish on Dental Caries in Chinese Preschool Children}

\section{INTRODUCTION}

n many developed countries, dental caries prevalence in children has decreased significantly in the past decades. The main reason for this decrease has been ascribed to improvement in oral hygiene, changes in lifestyles, use of fluoride, and implementation of school-based prevention programs (Marthaler et al., 1996). However, during the same time period, prevalence of dental caries has increased in many developing countries (Petersen, 2003). The second National Oral Health Survey conducted in China (Wang et al., 2002) found a high prevalence of caries in the primary dentition of five-year-old children, $77 \%$, and the mean number of decayed, missing, or filled primary teeth (dmft) was 4.5, with untreated active caries (dt) being the major component. Therefore, appropriate caries-preventive measures are urgently needed in China (Lin and Schwarz, 2001).

Chlorhexidine has been widely accepted as a chemotherapeutic agent against Streptococcus mutans, and studies have shown that chlorhexidine varnish can provide sustained release of chlorhexidine and suppress $S$. mutans in dental plaque for 6 mos after its application (Matthijs and Adriaens, 2002). Results of some clinical studies showed that chlorhexidine varnish is effective in caries prevention (Joharji and Adenubi, 2001; Baca et al., 2002). However, it has also been found to have little effect on new caries development (Fennis-Ie et al., 1998; Forgie et al., 2000; de Soet et al., 2002). Most of the reported studies were conducted on the permanent teeth of schoolchildren, and, in a recent review (Twetman, 2004), it was found that the evidence for the use of chlorhexidine varnish for caries prevention in risk groups was inconclusive. In a recent 24-month clinical trial on primary molars in six- to seven-year-old children (Baca et al., 2004), application of chlorhexidine varnish at three-monthly intervals resulted in a lower incidence of caries in children who were caries-free at baseline.

The purpose of this study was to assess the effect of six-monthly applications of $40 \%$ chlorhexidine varnish on the incidence of dental caries in primary molars in Chinese preschool children over a period of 2 yrs. The null hypothesis to be tested was that there was no difference in the new caries increment in primary molars between children who received chlorhexidine varnish every 6 mos and those who received a placebo varnish.

\section{MATERIALS \& METHODS}

This double-blinded, randomized, placebo-controlled clinical trial was carried out according to the guidelines for good clinical practice (MRC, 1998). The study protocol was reviewed and approved by the Ethics Committee of the School of Dentistry of the Wuhan University prior to the start of the study. Informed written consent was obtained from the parents of the children prior to the implementation of this study. The study was implemented in Hongshan
Received December 11, 2004; Last revision January 26, 2006; Accepted February 15, 2006 
Table. Mean dmfs-molar Scores of the Study Children in the Chlorhexidine and the Placebo Groups at Baseline and after 24 Months, and Their Caries Increments (SE in parentheses)

\begin{tabular}{lcccc}
\hline Group & $\mathrm{N}$ & $\begin{array}{c}\text { Mean dmfs-molar } \\
\text { at Baseline }\end{array}$ & $\begin{array}{c}\text { Mean dmfs-molar } \\
\text { at } 24 \text { Months }\end{array}$ & $\begin{array}{c}\text { Mean Caries } \\
\text { Increment, dmfs-molar }\end{array}$ \\
\hline CHX & 155 & $2.8(0.3)$ & $3.8(0.4)$ & $1.0(0.2)$ \\
Placebo & 135 & $\begin{array}{c}2.6(0.4) \\
{ }^{*} p=0.388\end{array}$ & $\begin{array}{c}4.2(0.4) \\
{ }^{*} p=0.224\end{array}$ & $\begin{array}{c}1.6(0.2) \\
{ }^{*} p=0.036\end{array}$ \\
\hline
\end{tabular}

* Two-sample $t$ test.

a dmfs-molar = the number of decayed, missing (due to caries), or filled primary molar surfaces. child was computed and used as the outcome measure. At the 24-month examination, soft tissues and all teeth in the mouth were examined for any possible side-effects, such as ulcers, other mucosal lesions, and tooth-staining. Duplicate examinations were carried out on one in ten children throughout the examinations.

All data were entered into a computer and analyzed by the software SPSS 11.0. We conducted two-sample $t$ tests to assess the differences between the two groups of children in their baseline dmfsmolar scores, and the new caries increments of their primary molars. The statistical significance level adopted was 0.05 .
District of Wuhan City, Hubei Province, in central China. The concentration of fluoride in the drinking water was 0.1-0.3 ppm, and no organized oral health care programs for preschool children were available in the study site.

We based our sample size calculation on ensuring our ability to detect a difference of 1 tooth surface in caries increment between the test group and the control group, if such a difference existed, at a statistical significance level of 5\% for a two-tailed, two-sample $t$ test, and at a power of $85 \%$. Results showed that a sample size of around 150 subjects in each group would be required.

Four kindergartens in the study district were chosen randomly in 1999, and all children aged 4-5 yrs in these kindergartens were invited to participate in the study. Informed written content was obtained from the parents of all children, and there were no refusals. We randomly divided the children into two groups, based on the class they attended, by drawing a card from a bag. Seven classes of children were assigned to the chlorhexidine varnish group (test), and seven classes were assigned to the placebo control group. The children, their parents, and the kindergarten staff were blinded as to the group assignment of the children.

The test material-a varnish that consisted of $40 \% \mathrm{w} / \mathrm{w}$ chlorhexidine acetate in a sandarach resin $(33 \% \mathrm{w} / \mathrm{w})$ dissolved in water-free alcohol $(27 \% \mathrm{w} / \mathrm{w})$ - was prepared in a laboratory by the investigators. The placebo varnish contained only an alcohol solution of sandarac. The 2 varnishes were put into bottles that had the same appearance, to ensure blindness of the trial. At the start of the study and every 6 mos thereafter, the chlorhexidine or the placebo varnish was applied to all surfaces of the primary molars of the children in the kindergartens, according to their group assignment, by two dentists who did not know the content of the varnish. No professional cleaning of teeth was carried out prior to varnish application. The teeth were isolated and dried with cotton rolls. The varnish was applied by means of a cotton swab and a sharp probe, and put on all tooth surfaces, including pits and fissures. The children were asked not to rinse, eat, and drink for 15 min after the varnish application.

Clinical examination took place at baseline (October, 1999) and after 24 mos (October, 2001). Two calibrated dentists who did not know the group assignment of the children performed all clinical examinations. The examinations were conducted in the kindergartens, with the use of mouth mirrors and probes under natural daylight. The status of each surface of the primary molars was recorded according to the criteria recommended by the World Health Organization (1997). The number of decayed, missing (due to caries), or filled primary molar surfaces (dmfs-molar) for each

\section{RESULTS}

In total, 334 children were originally included in the study175 in the test group and 159 in the placebo group. The ratio of boys to girls was 1:1.04. Results of the duplicate examinations showed that the inter-examiner reliability in assessing the tooth surface status was very good (Kappa $>0.90$ ). There was no significant difference between the baseline mean dmfs-molar scores of the two groups (2.8 vs. 2.6; $t$ test, $\mathrm{p}>0.05)$. After 2 yrs, 44 children (13\%) were lost to follow-up, because some (n = 31) had moved to other kindergartens, and some $(n=13)$ objected to the taste of the varnish and refused to be examined. The baseline mean dmfs-molar scores of the children who dropped out were similar to those of the children who remained in the study (2.6 vs. 2.7; $t$ test, $\mathrm{p}>0.05$ ).

The result presented below was based on the 290 children who remained in the study after 2 yrs (155 in the test group and 135 in the placebo group). The respective baseline mean dmfsmolar scores of these two groups of children were 2.8 and 2.6 (Table), and the difference was not statistically significant $(t$ test, $\mathrm{p}>0.05)$. The mean caries increment of the primary molars was $1.0 \mathrm{dmfs}-$ molar in the test-group children and 1.6 dmfs-molar in the placebo group. The difference of 0.6 tooth surface was equated to a $37.3 \%$ reduction in caries increment, and was statistically significant $(\mathrm{p}=0.036 ; 95 \% \mathrm{CI}=0.04-$ 1.16). No side-effects (such as soft-tissue lesions and staining of teeth) were found at the 24-month examination.

\section{DISCUSSION}

The main result of this study was a $37.3 \%$ reduction in new caries increment in the primary molars of the test-group children when compared with the control group over a two-year period. This clinical trial adopted a randomized, doubleblinded, placebo-controlled design. Although the random allocation of study children into the test and control groups on a class basis (cluster allocation) may not be ideal, this was adopted in this study to prevent error in painting the wrong varnish onto the teeth of the young children if children belonging to both groups were present in the same class. In all study kindergartens, the assignment of children to different classes was not done on any special basis, such as their demographic or socio-economic background. Thus, the children in the test and control groups were comparable. The excellent inter-examiner reliability found in this study may be taken to indicate that the amount of measurement bias in this study was small. 
The magnitude of caries reduction found in this study is comparable with that found in an earlier 24-month clinical trial (Baca et al. 2004). In that study, the percentage of reduction in decayed and filled primary molar surfaces was $11 \%$ and $46 \%$, respectively, for children with and without caries at baseline.

Studies that compared chlorhexidine varnish with topical fluoride applications (Petersson et al., 2000; Splieth et al., 2000) showed that they had similar effectiveness in preventing dental caries in the permanent teeth of teenagers. The $37.3 \%$ reduction in caries increment in this study is also close to that found in a meta-analysis of the results on effectiveness of fluoride varnish on caries prevention in primary teeth (Marinho et al., 2002), 33\% (95\% CI = 19-58\%).

Results of a two-year longitudinal study on a cohort of healthy schoolchildren, who received 3 applications of chlorhexidine varnish within a period of 2 wks, showed that suppression of caries progression was significantly related to the reduction of Streptococcus mutans levels (Twetman and Petersson, 1999). The caries-preventive effect of chlorhexidine varnish has also been shown to disappear 3 yrs after cessation of its regular application in schoolchildren (Baca et al., 2003).

So far, the published studies on the effectiveness of chlorhexidine varnish have focused on the prevention of dental caries in the permanent teeth of schoolchildren with high or low caries risk (Forgie et al., 2000; Splieth et al., 2000; Haukali and Poulsen, 2003). The present study is among the first to report on the effectiveness of chlorhexidine varnish in preventing caries in primary teeth in preschool children. Since both the prevalence and severity of dental caries in the primary molars in Chinese preschool children are very high, the positive result found in this study is very encouraging. The application of chlorhexidine varnish to tooth surfaces is a simple procedure and does not require a great deal of cooperation from the young children. It has the potential for wide-spread use in community prevention programs in China and in other parts of the world.

Based on the results of the study, the null hypothesis of this study was rejected. It is concluded that six-monthly applications of a $40 \%$ chlorhexidine varnish can prevent dental caries in the primary molars of preschool Chinese children.

\section{ACKNOWLEDGMENTS}

This study was supported by the National Key Technologies R \& D Program of the tenth Five-Year Plan, the Ministry of Science and Technology, and the National Committee for Oral Health (NCOH), People's Republic of China.

\section{REFERENCES}

Baca P, Munoz MJ, Bravo M, Junco P, Baca AP (2002). Effectiveness of chlorhexidine-thymol varnish for caries reduction in permanent first molars of 6-7-year-old children: 24month clinical trial. Community Dent Oral Epidemiol 30:363368.

Baca P, Junco P, Bravo M, Baca AP, Munoz MJ (2003). Caries incidence in permanent first molars after discontinuation of a school-based chlorhexidine-thymol varnish program. Community Dent Oral Epidemiol 31:179-183.
Baca P, Munoz MJ, Bravo M, Junco P, Baca AP (2004). Effectiveness of chlorhexidine-thymol varnish in preventing caries lesions in primary molars. J Dent Child 71:61-65.

de Soet JJ, Gruythuysen RJ, Bosch JA, van Amerongen WE (2002). The effect of 6-monthly application of $40 \%$ chlorhexidine varnish on the microflora and dental caries incidence in a population of children in Surinam. Caries Res 36:449-455.

Fennis-Ie YL, Verdonschot EH, Burgersdijk RC, König KG, van't Hof MA (1998). Effect of 6-monthly applications of chlorhexidine varnish on incidence of occlusal caries in permanent molars: a 3-year study. J Dent 26:233-238.

Forgie AH, Paterson M, Pine CM, Pitts NB, Nugent ZJ (2000). A randomised controlled trial of the caries-preventive efficacy of a chlorhexidine-containing varnish in high-caries-risk adolescents. Caries Res 34:432-439.

Haukali G, Poulsen S (2003). Effect of a varnish containing chlorhexidine and thymol (Cervitec) on approximal caries in 13to 16-year-old schoolchildren in a low caries area. Caries Res 37:185-189.

Joharji RM, Adenubi JO (2001). Prevention of pit and fissure caries using an antimicrobial varnish: 9 month clinical evaluation. $J$ Dent 29:247-254.

Lin HC, Schwarz E (2001). Oral health and dental care in modernday China. Community Dent Oral Epidemiol 29:319-328.

Marinho VC, Higgins JP, Logan S, Sheiham A (2002). Fluoride varnishes for preventing dental caries in children and adolescents. Cochrane Database Syst Rev 3:CD002279.

Marthaler TM, O'Mullane DM, Vrbic V (1996). The prevalence of dental caries in Europe 1990-1995. ORCA Saturday afternoon symposium 1995. Caries Res 30:237-255.

Matthijs S, Adriaens PA (2002). Chlorhexidine varnishes: a review. J Clin Periodontol 29:1-8.

Medical Research Council (MRC) (1998). MRC guidelines for good clinical practice in clinical trials. In: MRC Clinical Trials Series. London: External Communications.

Petersen PE (2003). The World Oral Health Report 2003: continuous improvement of oral health in the 21 st century-the approach of the WHO Global Oral Health Programme. Community Dent Oral Epidemiol 31(Suppl 1):3-23.

Petersson LG, Magnusson K, Andersson H, Almquist B, Twetman S (2000). Effect of quarterly treatments with a chlorhexidine and a fluoride varnish on approximal caries in caries-susceptible teenagers: a 3-year clinical study. Caries Res 34:140-143.

Splieth C, Steffen H, Rosin M, Welk A (2000). Caries prevention with chlorhexidine-thymol varnish in high risk schoolchildren. Community Dent Oral Epidemiol 28:419-423.

Twetman S (2004). Antimicrobials in future caries control? A review with special reference to chlorhexidine treatment. Caries Res 38:223-229.

Twetman S, Petersson LG (1999). Interdental caries incidence and progression in relation to mutans streptococci suppression after chlorhexidine-thymol varnish treatments in schoolchildren. Acta Odontol Scand 57:144-148.

Wang HY, Petersen PE, Bian JY, Zhang BX (2002). The second national survey of oral health status of children and adults in China. Int Dent J 52:283-290.

World Health Organization (1997). Oral health survey-basic methods. 4th ed. Geneva: World Health Organization. 